\title{
Análise de deformações específicas de tração na fibra inferior de revestimento asfáltico através de instrumentação e métodos numéricos
}

\author{
Nielson Tôrres Neves de Carvalho' ${ }^{1}$, Fernando Silva Albuquerque ${ }^{2}$ \\ ${ }^{1}$ Laboratório de Topografia e Transportes, Departamento de Engenharia Civil, UFS, nielsontorres@gmail.com \\ ²Laboratório de Topografia e Transportes, Departamento de Engenharia Civil, UFS, albuquerque.f.s@uol.com.br
}

\section{Recebido:}

1 de fevereiro de 2018

Aceito para publicação:

18 de julho de 2019

Publicado:

31 de agosto de 2019

Editor de área:

Jorge Barbosa Soares

\section{Palavras-chaves:}

Instrumentação,

Modelos numéricos,

Deformação de tração.

\section{Keywords:}

Instrumentation,

Numerical models,

Tensile strain.

DOI:10.14295/transportes.v27i2.1579

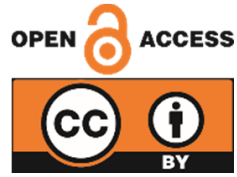

\begin{abstract}
RESUMO
O uso de estimativas numéricas das tensões, deformações e deflexões resultantes do tráfego de veículos, para dimensionamento de pavimentos rodoviários, é bastante comum pela praticidade, porém pode trazer imprecisões, provocando superdimensionamento ou subdimensionamento das camadas do pavimento. Por sua vez, o uso da instrumentação, realizando medições diretas no pavimento, tende a fornecer maior exatidão. Dentre as respostas mecânicas críticas, destaca-se a deformação específica de tração na fibra inferior das camadas asfálticas, ligada ao processo de fadiga. Neste trabalho foi realizada avaliação dessa deformação medida através de sensor H-Gage, comparando em seguida com as estimativas numéricas através de modelagem com os métodos das múltiplas camadas elásticas e dos elementos finitos. Ambos os modelos numéricos apresentaram estimativas bem discrepantes das medições de campo, tendo diferença ainda maior nas respectivas previsões de vida de fadiga. O método das múltiplas camadas elásticas apresentou tendência a estimar menor vida de fadiga, enquanto o modelo proposto em elementos finitos tendeu a estimar maior vida de fadiga, à medida que se reduzia o atrito entre camadas.
\end{abstract}

\begin{abstract}
Numerical analyzes of stress, strains and displacements resulting from vehicle traffic for pavement designs is usual, but may lead inaccuracies, causing oversizing or undersizing of pavement layers. On the other hand, the measurement of data by instrumentation on the pavement tends to provide greater accuracy. Among the main mechanical responses, the critical tensile strain on the bottom of the asphalt layers is related to the fatigue process. In this work, it was carried out a measurement of the tensile strain by the $\mathrm{H}$-Gage sensor, comparing to the numerical analyzes by modeling the elastic multilayers and finite element methods. Both numerical models presented very different estimates of the wearing course measurements, where the greater difference was in the fatigue life predictions. The elastic multilayers method tended to estimate shorter fatigue life, while the proposed finite element model tended to estimate longer fatigue life, as the friction between layers was reduced.
\end{abstract}

\section{INTRODUÇÃO}

Os métodos numéricos são as principais fontes de estimativa de tensões, deformações e deslocamentos dos pavimentos, para uso em projeto e gerenciamento de pavimentos, destacando-se o método das múltiplas camadas elásticas e o Método dos Elementos Finitos (MEF). Já a instrumentação com sensores utilizada para calcular deslocamentos, deformações, tensões, e variáveis ambientais como temperatura, pode inferir resultados mais exatos com medições diretas no pavimento (DEUSEN et al., 1992). 
Várias pesquisas analisaram o comportamento mecânico de pavimentos asfálticos através de instrumentação e de modelagens numéricas. Saevarsdottir e Erlingsson (2016) verificaram boa compatibilidade entre elas, Dong et al. (2012) verificaram diferenças de 3\% a 7\% para tensões verticais e $18 \%$ a $30 \%$ para deformações específicas horizontais de tração comparando resultados de sensores e estimativas numéricas, Bastos (2016) analisou performance de pavimento através de instrumentação e ensaios de laboratório, confirmando efetividade da instrumentação para identificar processos de degradação do pavimento, Matos (2015) realizou análise de tensões verticais em pista experimental através de instrumentação e modelo numérico, encontrando diferença média de 38,5\%, já Leiva-Villacorta e Timm (2012) atestaram a importância da locação dos sensores e do tipo material de recomposição utilizado para a eficiência da instrumentação.

Diante desse fato, levanta-se a seguinte questão: Resultados numéricos são compatíveis com os de instrumentação? Para responder essa pergunta, foi realizada análise das deformações específicas de tração na fibra inferior de camada de revestimento de concreto asfáltico, obtidas com instrumentação e com os métodos numéricas de sistema de múltiplas camadas elásticas e de elementos finitos, avaliando suas diferenças e os impactos nas previsões de vida de fadiga.

\section{FADIGA EM CAMADAS ASFÁLTICAS}

Um dos mais importantes processos de degradação de camadas cimentadas de pavimentos (asfálticas ou outro agente cimentante), a fadiga, é um processo cumulativo provocado pela repetição de deformações de tração horizontal na fibra inferior da camada, forma fissuras e pode levar ao rompimento da camada (TEIXEIRA et al., 2007). 0 número de ciclos de carregamento suportado pela camada (vida de fadiga) é estimado através de modelo de previsão de desempenho adequado (SAPEM, 2014).

0 processo de fadiga apresenta uma evolução diferente, a depender das magnitudes de carregamentos que solicitam as camadas cimentadas. Disto decorre que cada um destes diferentes carregamentos será responsável por parcelas do dano acumulado por fadiga ao material, resultando em trincamentos na camada (FRITZEN, 2016). Isso torna o problema de difícil resolução, especialmente pelas incertezas de como as cargas de tráfego realmente agem nos pavimentos, da variação da rigidez das camadas (especialmente as asfálticas) de acordo com a temperatura do material e frequência de carregamento, de como as camadas interagem umas com as outras (existência de aderência ou atrito), entre outros (KIM et al., 2008; NASCIMENTO, 2015; FRITZEN, 2016).

Especialmente sobre o atrito, esta teoria foi desenvolvida por Charles-Augustin de Coulomb em 1785. Ele investigou a influência de quatro fatores principais no atrito: a natureza dos materiais em contato e seus revestimentos de superfície; a extensão da área de superfície; a pressão normal (ou carga); e o tempo que as superfícies permaneceram em contato (tempo de repouso). Ainda definiu que o atrito não é em si uma força fundamental. 0 atrito a seco surge de uma combinação de aderência entre superfícies, rugosidade da superfície, deformação da superfície e contaminação da superfície (MADENCI et al., 2006; NAKASONE et al., 2006).

Em análises de sólidos elastoplásticos, a maior parte da energia do atrito na interface de sólidos é dissipada através da deformação num grande volume ao redor da área de contato, até que se atinja a tensão de cisalhamento máxima. Ainda, a maior parte dessa deformação tende a ser plástica, com mudança permanente de forma sem fratura. No que se refere à deformação plástica causada devido ao atrito na interface de sólidos, há uma tendência ao crescimento à 
medida que o coeficiente de atrito também aumente (LI et al., 2010; PELLETIER et al., 2011; TRZEPIECIŃSKI et al., 2015).

No caso de sólidos elásticos, a maior parte da energia entre as camadas com atrito é de adesão (LI et al., 2010; PELLETIER et al., 2011; TRZEPIECIŃSKI et al., 2015), fazendo com que elas trabalhem de forma monolítica, provocando menor esforço de flexão e, consequentemente, menores deformações e maior vida de fadiga, destacando que esse comportamento depende também dos tipos e rigidezes das camadas envolvidas (BALBO, 2007; SILVA et al., 2015). Silva et al. (2015) verificaram, através de simulações numéricas de camadas elásticas, forte influência da aderência entre camadas asfálticas, observando queda de vida de fadiga em dezoito anos para situação sem aderência comparada com aderência total, destacando também a dificuldade para estimar a aderência em campo.

Portanto, a condição de atrito mais apropriada ao propósito de análise, associada ao comportamento mecânico mais aproximado do material, deve ser selecionada para o êxito na condução da análise.

\section{MÉTODOS NUMÉRICOS PARA ANÁLISE DE PAVIMENTOS}

O grau de complexidade do comportamento mecânico dos pavimentos limita a utilização de soluções analíticas para seu entendimento, sendo mais viáveis soluções numéricas (FONSECA et al., 2002). Por outro lado, estimativas numéricas contêm imprecisões decorrentes de hipóteses simplificadoras utilizadas para facilitar os cálculos, que inclusive são esperadas e comprovadas, conforme pesquisas que fizeram comparações com medidas diretas no pavimento, oriundas da instrumentação (GONÇALVES, 2002; DONG et al., 2012; SAEVARSDOTTIR e ERLINGSSON, 2016).

O Método das Múltiplas Camadas Elásticas, baseado na Teoria do Sistema de Múltiplas Camadas Elásticas, desenvolvido por Burmister em 1945 e com fundamento na teoria da elasticidade e nas equações de Boussinesq (1885) para um sólido semi-infinito, considera os elementos do pavimento ou solo estratificado como camadas que respeitam hipóteses simplificadoras, considerando o pavimento como uma estrutura de camadas sobrepostas (BURMISTER, 1945a; BURMISTER, 1945b).

Nesse modelo, em geral, o problema é solucionado com métodos numéricos computacionais, utilizando equações limites, relação deformação/deslocamento, características constitutivas e mecânicas dos materiais das camadas, e condições contorno (IOANNIDES e KHAZANOVICK, 1998). Ele possibilita o calculado isolado do estado tridimensional de tensões em qualquer ponto do pavimento. Franco (2007) desenvolveu software chamado SisPav, que utiliza esse método, através do módulo AEMC (Análise Elástica de Múltiplas Camadas), para calcular respostas mecânicas do pavimento e realizar dimensionamento de camadas através de modelos mecanísticos-empíricos. A grande limitação deste sistema na versão de teste disponibilizada é de fornecer apenas soluções elástico-lineares para materiais asfálticos, quando é sabido que o comportamento desta camada é dependente da temperatura do material e da frequência de carregamento.

Baseado em aproximações numéricas para solucionar equações diferenciais por integração através de condições de contorno generalizadas, o MEF consiste em dividir o meio contínuo (chamado de domínio) em partes menores, chamadas de elementos finitos, que têm inerentes funções que descrevem variáveis de campo e são ligados entre si através de nós. Isso torna 
possível o cálculo dos estados de tensão para todo domínio, conforme condições iniciais de carregamento externo e de restrições ao deslocamento (MADENCI e GUVEN, 2006).

Na pavimentação, o MEF se destaca pela possibilidade de utilização de diferentes modelos constitutivos e de formação e propagação de danos (SOUZA e SOARES, 2003). NCHRP/TRB (2004) traz orientações para melhorar a eficácia do MEF, como realizar maior discretização do domínio nas camadas mais próximas do carregamento e de maior interesse de estudo (maior número de elementos finitos) e menor nas camadas mais profundas ou de pouco interesse (menor número de elementos finitos e, consequentemente, menor esforço computacional), e utilizar domínio com dimensões horizontais suficientes para que as suas faces verticais distem do centro da carga de 10 (dez) a 12 (doze) vezes o raio da mesma e dimensão vertical de no mínimo 50 (cinquenta) vezes o raio da carga.

A utilização do MEF é viabilizada através do uso de softwares, a exemplo do ANSYS®, bastante utilizado para soluções mecânicas em várias áreas da engenharia, e o CAP3D, desenvolvido no Laboratório de Mecânica dos Pavimentos - LMP da Universidade Federal do Ceará UFC, com objetivo de modelagem de pavimentos (HOLANDA et al., 2006).

\section{INSTRUMENTAÇÃO EM PAVIMENTOS}

A instrumentação de pavimentos traz a possibilidade de acompanhar reações mecânicas de acordo com variações das propriedades das camadas e de variáveis ambientais, fornecendo melhor entendimento do comportamento do pavimento (DEUSEN et al., 1992; DNIT, 2008).

Existem alguns tipos de sensores utilizados na instrumentação de pavimentos, e a escolha deve levar em conta os seguintes fatores (ELSEIFI et al., 2012):

- Tipo de resposta (maior parte em diferença de potencial elétrico);

- Sensibilidade de resposta;

- Compatibilidade do mecanismo do sensor com o sistema de aquisição de dados;

- Expectativa de vida;

- Limitações referentes à temperatura e eletromagnetismo;

- Tipo de camada a ser instrumentada; e

- Possível interferência entre os sensores utilizados em mesma seção.

Na pavimentação existem vários tipos de sensores, como células de carga ou de pressão para medição de tensão, termopares para medição de temperatura e extensômetros (strain gages) para medição de deformações. Nos extensômetros, utilizados neste trabalho, as medições de deformação são baseadas na correlação com a variação da resistência elétrica.

A mudança de resistência elétrica devido às deformações é muita pequena e de difícil leitura, que é possibilitada através de circuito eletrônico composto de quatro extensômetros chamado de ponte de Wheatstone, podendo ser modificada para $1 / 2$ ponte ou $1 / 4$ de ponte, substituindo dois ou três extensômetros por resistores de mesma resistência elétrica, respectivamente.

A temperatura e o efeito eletromagnético, provocado pelo próprio circuito ou por fontes externas, são fatores ambientais que influenciam as respostas elétricas dos sensores e, consequentemente, a eficácia. A temperatura altera dimensões dos extensômetros e, consequentemente, a resistência elétrica. Já as ondas eletromagnéticas geram flutuações de tensão elétrica no circuito. A ponte de Wheatstone e modificações supracitadas também ajudam 
a mitigar os efeitos desses fatores, promovendo compensação de temperatura entre sensores e eliminando influência de temperatura nos cabos (DEUSEN et al., 1992; KYOWA, 2011).

\section{MÉTODOS DE TRABALHO}

\subsection{Pista experimental}

O objeto da análise de deformações foi a pista experimental "A", localizada no Campus de São Cristóvão, da Universidade Federal de Sergipe (PEA-UFS). Essa pista é composta de camada de revestimento em concreto asfáltico (CA) de 7,0 cm, base antirreflexão de trincas em solo arenoargiloso de 7,0 cm (solo A-2-6 - AASHTO), sub-base em solocimento (4\%) de 25,0 cm (solo A2-4 - AASHTO + 4\% de cimento Portland CPII-Z-32-RS) e com resistência à compressão simples aos 7 (sete) dias de 4,15 MPa, reforço de subleito em solo arenoargiloso de 7,0 cm (solo A-2-6 AASHTO), e subleito em solo arenoso confinado, como representado na Figura 1a.

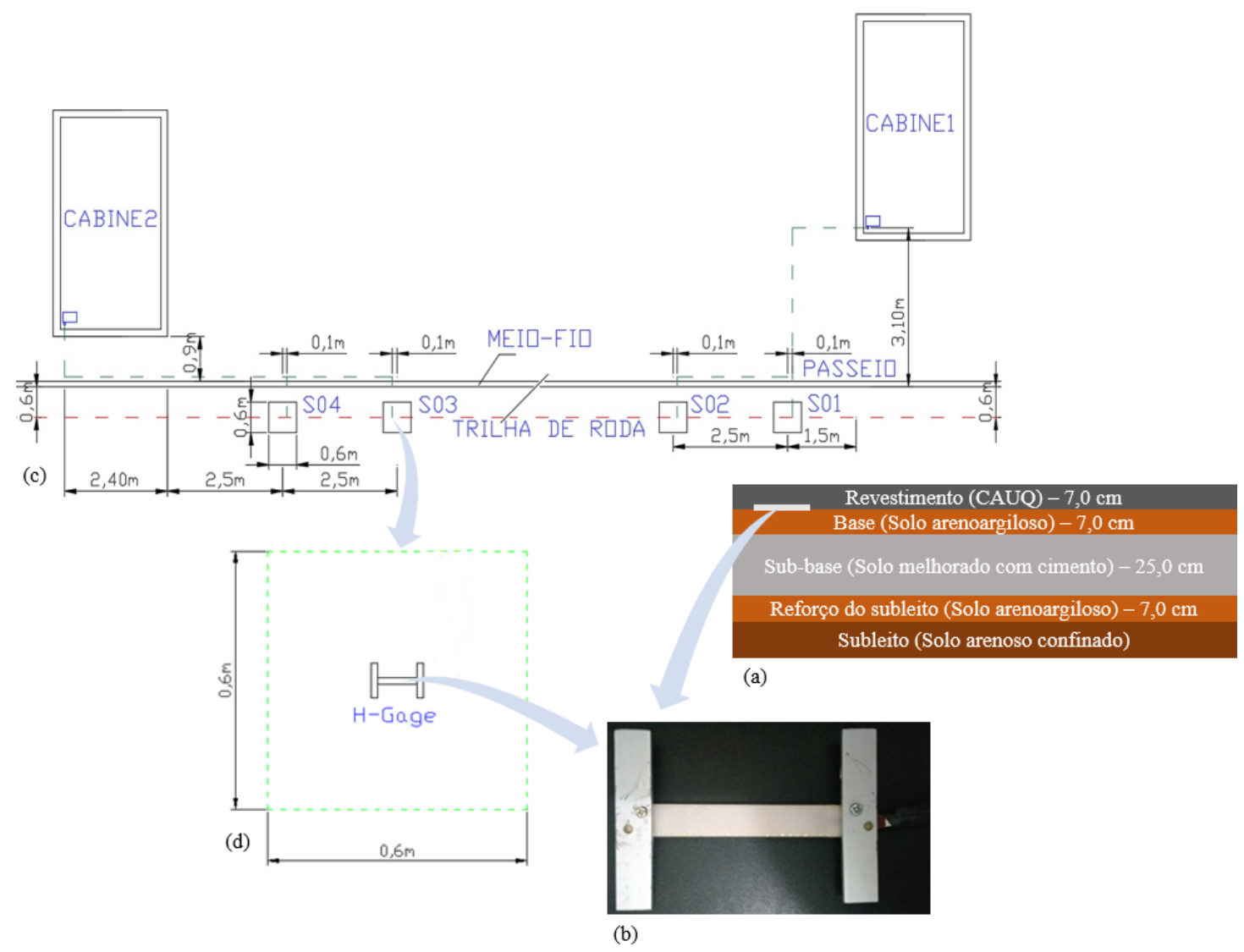

Figura 1. (a) Perfil do pavimento asfáltico; (b) H-Gage desenvolvido utilizado; (c) Esquema de instalação dos sensores nas seções da pista experimental; (d) Esquema de locação do sensor H-Gage em seção tipo.

A execução do pavimento foi acompanhada por Matos (2015), quando foi realizada caracterização das camadas do pavimento, obtendo-se seus Módulos de Resiliência (MR) através de ensaio de tração indireta com carregamento repetitivo para a mistura asfáltica, triaxial com carregamento repetitivo para a base e o subleito, carregamento uniaxial dinâmico para a sub-base e retroanálise de bacias deflectométricas somente para o subleito (Tabela 1). Os valores de coeficiente de Poisson $(\mu)$ das camadas foram estimados de acordo com a bibliografia consultada (BERNUCCI et al., 2010). 
Tabela 1: Propriedades mecânicas das camadas do pavimento (Matos, 2015)

\begin{tabular}{cccc}
\hline Camadas & MR (MPa) & $\mathbf{R}^{\mathbf{2}}$ & $\mu$ \\
\hline Revestimento & $4.880,73$ & & 0,25 \\
Base & $M R=188,88 . \sigma_{3}^{0,1905}$ & 0,82 & 0,30 \\
Sub-base & $M R=6998,50 \sigma_{d}^{0,664}$ & 0,96 & 0,25 \\
Reforço do subleito & $M R=272,60 \theta^{0,430}$ & 0,89 & 0,40 \\
Subleito' & 110,00 & & 0,30 \\
\hline
\end{tabular}

$\begin{array}{lll}\text { em que } & \sigma_{3}: & \text { tensão de confinamento [MPa]; } \\ & \sigma_{d}: & \text { tensão desvio [MPa]; e } \\ & \theta: & \text { primeiro invariante de tensões [MPa]. }\end{array}$

\subsection{Instrumentação e Caracterização da Pista Experimental}

A primeira etapa desta pesquisa foi desenvolver os sensores para medição das deformações na fibra inferior do revestimento, denominados H-Guages. Eles foram montados utilizando extensômetro de imersão de 12,0 cm e duas ancoragens em alumínio de 8,0 cm. 0 sensor H-Gage desenvolvido na pesquisa pode ser visto na Figura $1 \mathrm{~b}$.

0 circuito eletrônico utilizado foi equivalente à meia ponte de Wheatstone composto de dois sensores. Essa composição fornece compensação de temperatura nos sensores e nos cabos, sendo as deformações calculadas com base na Equação 1 (KYOWA, 2011):

$$
\frac{e_{0}}{E}=F_{G} \cdot \frac{\varepsilon}{4}
$$

$\begin{array}{rll}\text { em que } & e_{0}: & \text { tensão elétrica de saída do circuito }[\mathrm{mV}] \\ & E: & \text { tensão elétrica de entrada do circuito }[\mathrm{mV}] ; \\ & F_{G}: & \text { fator gage, neste caso igual a 2,0; e } \\ & \varepsilon: & \text { deformação equivalente }[\mathrm{m} / \mathrm{m}] .\end{array}$

Esses sensores foram instalados em quatro seções do pavimento (S01 a S04) (Figura 1c), e ainda foram instaladas sondas termopar tipo "K" em seção representativa para medição de gradiente de temperatura em três profundidades do revestimento. Para a instrumentação foi necessário realizar demolição do revestimento até a altura do topo da base, com abertura de janelas de 0,6 x 0,6 m (Figura 1d), e recomposição posterior, que foi realizada utilizando concreto asfáltico com aditivo polimérico, que possibilitou a execução do novo revestimento horas após a sua fabricação.

O novo revestimento e o revestimento original do pavimento passaram por caracterização mecânica através de ensaios de massa específica aparente, resistência à tração indireta (RTI), conforme norma DNIT 031/2016-ES, e ensaio de ressonância por impacto (RI), conforme norma ASTM E1876 (2009) e modelos de shift factor definidos por Costa (2017). Os corpos de prova utilizados nesses ensaios foram extraídos do pavimento após a etapa de medições de campo e retificados nas dimensões exigidas pelas normas.

No ensaio de RI foram geradas curvas mestras do módulo dinâmico $\left|E^{*}\right|$ em função da frequência de carregamento reduzida $(f)$ na temperatura de referência $(\mathrm{t})$ inicial de $20^{\circ} \mathrm{C}$ e depois extrapolada para a temperatura padrão de $40^{\circ} \mathrm{C}$. A escolha desta temperatura foi por ser aproximadamente a média observada nas seções em todos os três dias de medições de campo, no 
período T1, obtendo-se, para frequência reduzida teórica de $1 \mathrm{~Hz}$, valores de $\left|\mathrm{E}^{*}\right|$ e de $\mu$ para os materiais utilizados na recomposição e para a mistura original. Destaca-se que o valor de MR e do $\mu$ originais da mistura asfáltica (Tabela 1) não foram utilizados nas simulações numéricas, preferindo-se utilizar o $\left|E^{*}\right|$ e o $\mu$ obtidos no ensaio de RI, que são mais coerentes com as condições de temperatura de campo.

\subsection{Medições na Pista Experimental}

Após instrumentação da pista, foram medidas tensões elétricas registradas nos sensores e obtidas, indiretamente, as deformações em campo para três magnitudes de cargas diferentes no eixo traseiro simples de roda dupla de veículo comercial do tipo caminhão caçamba. A primeira carga foi aproximadamente 8,20 t, carga do eixo padrão, a segunda entre 10,00 e 11,00 t, faixa de tolerância para esse tipo de eixo nas rodovias brasileiras segundo resoluções $n^{\circ} 12 / 1998$ e no 526/2015 do CONTRAN, e a terceira acima de 11,00 t, ou seja, fora do permitido. Para cada carregamento, foram realizadas medições em dois horários diferentes para simular faixas de temperatura também diferentes, uma pela manhã (T1) e outra pela tarde (T2), em cada uma das quatro seções.

Foram realizadas três leituras com passagem do semieixo de roda dupla do caminhão caçamba para cada tipo de carregamento e faixa de temperatura, buscando alinhamento do ponto médio entre as duas rodas (posição da resultante da carga do semieixo) e a posição do sensor que foi previamente marcada no revestimento. 0 veículo era previamente posicionado de forma que o semieixo estivesse alinhado com a posição do sensor, mas com distância suficiente para não haver deformação no sensor, e em seguida, acompanhando as leituras em tempo real era realizada a passagem do semieixo sobre a seção instrumentada de forma quase-estática (velocidade média abaixo de $5 \mathrm{~km} / \mathrm{h}$ ) no sentido longitudinal ao tráfego, realizando leitura do pico de variação da relação de tensão elétrica $\left(e_{0} / E\right)$, e, em seguida, calculando-se a respectiva deformação através da Equação 1.

As amostras de campo passaram por tratamento estatístico utilizando o critério de Chauvenet para levantar possíveis dados discrepantes e eliminá-los do cálculo da deformação média. Em seguida foi ajustado modelo fatorial linear completo das deformações, com objetivo exclusivo de analisar influência dos níveis de carregamento (fator "C") e de temperatura (fator "T") nos valores obtidos através da instrumentação. Esse modelo é mostrado na Equação 2:

$$
\varepsilon=b_{0}+b_{1} \cdot T+b_{2} \cdot C+b_{3} \cdot T \cdot C
$$

$$
\begin{aligned}
& \text { em que } \varepsilon \text { : deformação específica de tração }[\mathrm{m} / \mathrm{m}] \text {; } \\
& b_{0}, b_{1}, b_{2} \text { e } b_{3} \text { : coeficientes do modelo obtidos através de regressão linear; } \\
& \text { T: nível de temperatura; e } \\
& \text { C: nível de carregamento. }
\end{aligned}
$$

Nessa análise as probabilidades dos estimadores (coeficientes $b_{0} \mathrm{a} b_{3}$ ) deveriam ser inferiores a $5 \%$ para exercer influência na inferência de $\varepsilon$. A análise fatorial foi complementada com o cálculo da variação percentual das medições com a variação de magnitude de carregamento e temperatura, verificando a influência desses dois fatores na deformação e se houve algum comportamento anormal. 


\subsection{Análises Numéricas}

Obtidos os parâmetros de rigidez dos revestimentos, foram estimadas deformações horizontais no ponto crítico do revestimento, na sua fibra inferior e alinhado à posição da resultante central do carregamento do semieixo, ou seja, em posição equivalente ao local de instalação dos sensores, através dos métodos das múltiplas camadas finitas e dos elementos finitos. Em ambos os métodos foram realizadas análises para temperatura de referência de $40^{\circ} \mathrm{C}$, utilizando características elásticas das camadas do pavimento semelhantes às reais, assim como magnitudes dos carregamentos externos.

Para o primeiro método foi utilizado o software AEMC (2.0.1.1), considerando como revestimento o de recomposição e desconsiderando o antigo adjacente às seções. Nessa análise foram utilizadas as características viscoelásticas $\left(\left|E^{*}\right|\right.$ e $\mu$ ) encontradas através do ensaio de RI para o revestimento das seções e os encontrados por Matos (2015) para as demais camadas (MR e $\mu$ ). Já o carregamento externo foi equivalente aos de campo, inclusive a área de contato pneu-pavimento. A aderência entre a camada de revestimento e de base não foi considerada, pois, neste caso, não forneceu diferença significativa nas deformações estudadas. Através da análise no AEMC/SisPav, também foram obtidos os estados de tensão das camadas de base, sub-base e reforço de subleito, necessários para linearização dos módulos de resiliência destas camadas que foram utilizados na modelagem em elementos finitos.

A análise em elementos finitos foi realizada através do software ANSYS® Academic Teaching Introductory (Release 17.0). O ANSYS ${ }^{\circledR}$ possibilitou levar em conta não só o revestimento de recomposição, mas também o original adjacente. 0 modelo desenvolvido tem domínio representativo do pavimento de 1,70 x 2,20 m na horizontal por 5,40 m na vertical, conforme orientação do NCHRP/TRB (2004), considerando carga circular teórica de 10,80 cm de raio. Respeitando o limite disponível desta versão do software, a maior discretização foi no revestimento de recomposição e no alinhamento da carga, utilizando elementos finitos hexaédricos, com oito nós de três graus de liberdade cada, do tipo SOLID 185. Esse elemento é caracterizado por ter plasticidade, hiperelasticidade, fluência, grande deflexão e grandes capacidades de deformação, com capacidade para análises elastoplásticas (MADENCI et al., 2006; NAKASONE et al., 2006).

Para todas as camadas do pavimento no modelo em elementos finitos, foram atribuídos modelos de comportamento lineares. Para os dois tipos de revestimento foram utilizados os módulos dinâmicos obtidos no ensaio de RI e para as demais camadas os módulos de resiliência linearizados. As análises foram realizadas para magnitudes de carregamento iguais às de campo, porém com área de contato pneu-pavimento padronizada, 20,00 x 30,00 cm para os três tipos, devido a restrições da discretização do modelo, e considerando ou não o coeficiente de atrito $(0,0 ; 0,7 ;$ e 1,0$)$ entre o novo revestimento e a base, coeficiente de atrito $(0,0 ; 0,7 ;$ e 1,0$)$ entre a camada de revestimento novo e o antigo, e nenhum atrito nas demais interfaces entre camadas.

\subsection{Validação dos Modelos de Análise e Avaliação de Desempenho de Pavimentos}

Realizadas as análises numéricas, as estimativas foram comparadas entre si e com os valores da instrumentação, levantando qual método fornece valores próximos aos de campo. De forma semelhante, foi realizado comparativo para as respectivas previsões de fadiga calculadas através de modelo do South African Mechanistic Design Method (SAPEM, 2014) mostrado na Equação 3, 
tomando como referência de exatidão os valores da instrumentação e verificando possíveis consequências do uso dos modelos numéricos:

$$
N_{f}=10^{\alpha}\left(1-\frac{\log \varepsilon}{\beta}\right)
$$

em que $\quad N_{f}$ : $\quad$ vida de fadiga em repetição da passagem de eixo padrão de 8,20 t;

$\varepsilon$ : deformação específica de tração na fibra inferior do revestimento asfáltico $[\mathrm{m} / \mathrm{m}] ; \mathrm{e}$

$\alpha$ e $\beta$ : $\quad$ constantes que dependem do tipo de camada asfáltica e nível de confiança do projeto ou tipo de via.

0 pavimento estudado, projetado para um período de 20 anos, com repetições do eixo padrão

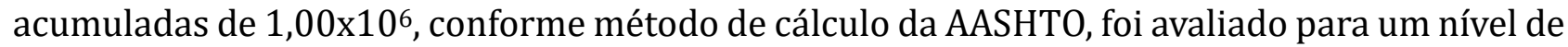
significância de 50\% (nível de confiança D), enquadrando-se nas características de vias coletoras, tendo então as constantes $\alpha$ e $\beta$ os valores de 17,71 e 3,46, respectivamente (SAPEM, 2014).

\section{RESULTADOS E DISCUSSÃO}

\subsection{Medidas de Deformações Específicas de Tração}

As medições de campo foram realizadas com carregamentos de 8,27 t, 10,47 t e 11,54 t, devidamente medidos em balança aferida. As áreas de contato, o espaçamento entre pneus e as temperaturas médias para as três magnitudes de carregamento medidas no período da manhã (temperatura T1) e no período da tarde (temperatura T2) podem ser vistas na Tabela 2, verificando-se que as temperaturas em T1 para os três carregamentos foram próximas.

Tabela 2: Áreas de contato e temperaturas medidas em campo por carregamento

\begin{tabular}{ccccc}
\hline \multirow{2}{*}{ Carregamento $(\mathbf{t})$} & \multicolumn{2}{c}{ Temperaturas } & \multicolumn{2}{c}{ Contato pneu-pavimento } \\
\cline { 2 - 5 } & $\mathbf{T 1}\left({ }^{\circ} \mathbf{C}\right)$ & $\mathbf{T 2}\left({ }^{\circ} \mathbf{C}\right)$ & Área de Contato $\left(\mathbf{c m}^{2}\right)$ & Espaçamento entre pneus $(\mathbf{c m})$ \\
\hline 8,27 & 38,50 & 44,43 & $23,0 \times 26,0$ & 11,0 \\
10,47 & 40,40 & 43,00 & $23,0 \times 28,0$ & 10,0 \\
11,54 & 40,80 & 38,80 & $23,0 \times 30,0$ & 10,0 \\
\hline
\end{tabular}

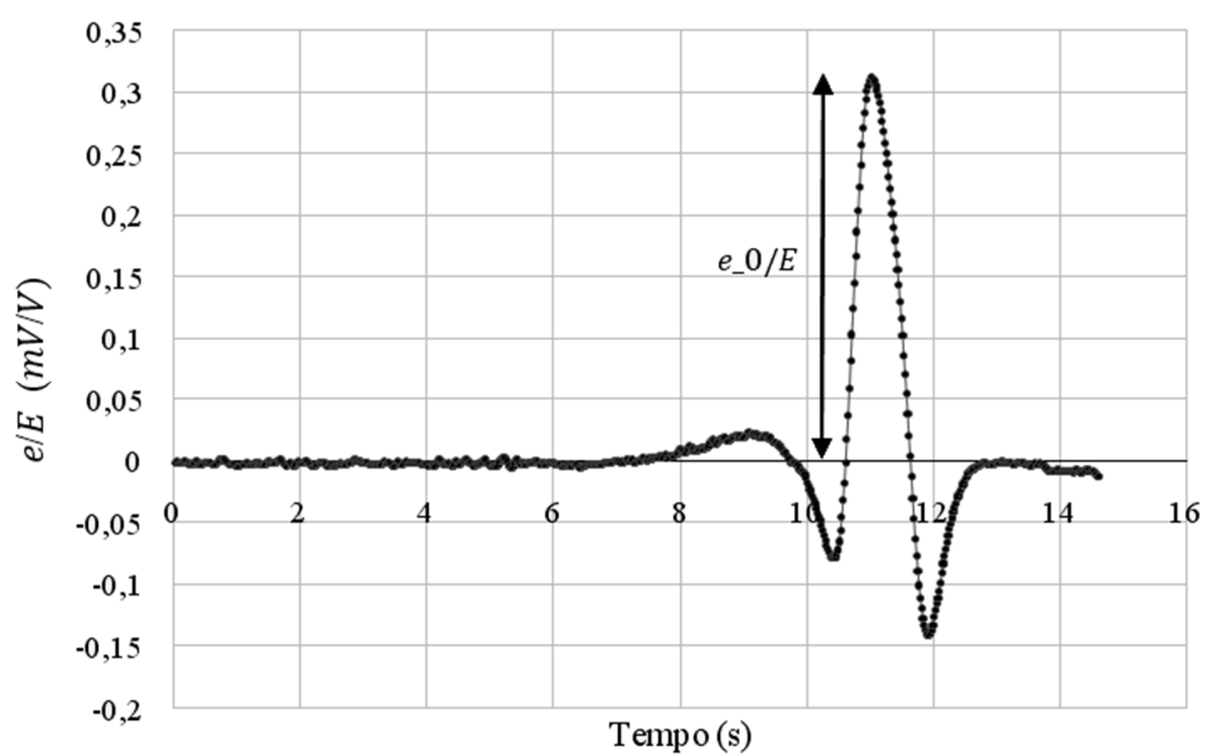

Figura 2. Pulso de variação da relação de tensão elétrica com o tempo no H-Gage na primeira repetição da medição com 11,54 t, na seção 02 e temperatura T1. 
Cada medição gerou pulsos de variação da relação entre tensão elétrica de saída $(e)$ e de entrada $(E)$ no circuito do H-Gage e o tempo, tendo o seu pico $\left(e_{0} / E\right)$ no momento da passagem do carregamento sobre o sensor. A Figura 2 apresenta um pulso característico da primeira réplica da medição com o carregamento de 11,54 t, na seção 02 e temperatura T1, exemplificando o processo de aquisição de dados, onde pode ser visto ainda pulsos de deformação de compressão antes e após o pico de tração

0 pico de variação da relação de tensão elétrica foi utilizado para calcular a deformação específica equivalente no $H$-Gage através da Equação 1, em cada uma das réplicas de medição, passando posteriormente essas deformações por tratamento estatístico, onde nenhum dado foi eliminado em princípio. No caso do pulso da Figura 2, o pico foi de 0,312 mV/V, e consequentemente a deformação foi calculada em $0,000264 \mathrm{~m} / \mathrm{m}$.

A Tabela 03 apresenta, para cada medição, as magnitudes de carregamento, a temperatura e a deformação específica média de tração das réplicas com suas respectivas incertezas relativas, que foram altas (>20,00\%) em algumas medições. Também são apresentadas as variações da deformação com a temperatura $\left(\Delta \varepsilon /{ }^{\circ} \mathrm{C}\right.$, de T1 para T2) e as variações de deformação com a mudança de magnitude de carga na temperatura padrão (T1).

Tabela 2: Áreas de contato e temperaturas medidas em campo por carregamento

\begin{tabular}{|c|c|c|c|c|c|c|c|c|c|}
\hline \multirow[t]{2}{*}{$P(t)$} & \multirow[b]{2}{*}{ Seção } & \multicolumn{3}{|c|}{$\mathrm{T} 1$} & \multicolumn{3}{|c|}{$\mathrm{T} 2$} & \multirow{2}{*}{$\begin{array}{c}\Delta \varepsilon /{ }^{\circ} \mathrm{C} \\
(\%)\end{array}$} & \multirow{2}{*}{$\begin{array}{c}\Delta \varepsilon /{ }^{\circ} \mathrm{C} \text { média } \\
\text { (\%) }\end{array}$} \\
\hline & & Medição & $\varepsilon(\mathrm{m} / \mathrm{m})$ & $\begin{array}{c}\text { Incerteza } \\
\text { Relativa (\%) }\end{array}$ & Medição & $\varepsilon(\mathrm{m} / \mathrm{m})$ & $\begin{array}{c}\text { Incerteza } \\
\text { Relativa (\%) }\end{array}$ & & \\
\hline \multirow{4}{*}{8,27} & S01 & 1 & 0,000680 & 9,45 & 13 & 0,000970 & 6,65 & $43 \%$ & \multirow{4}{*}{$108 \%$} \\
\hline & S02 & 2 & 0,000190 & 22,42 & 14 & 0,000590 & 22,09 & $211 \%$ & \\
\hline & S03 & 3 & 0,000370 & 8,44 & 15 & 0,000760 & 3,77 & $105 \%$ & \\
\hline & SO4 & 4 & 0,000430 & 22,60 & 16 & 0,000740 & 6,71 & $72 \%$ & \\
\hline \multirow{4}{*}{10,47} & S01 & 5 & 0,000400 & 4,98 & 17 & 0,000780 & 7,55 & $95 \%$ & \multirow{4}{*}{$24 \%$} \\
\hline & S02 & 6 & 0,000500 & 8,73 & 18 & 0,000570 & 13,96 & $14 \%$ & \\
\hline & S03 & 7 & 0,000390 & 10,42 & 19 & 0,000310 & 22,25 & $-21 \%$ & \\
\hline & SO4 & 8 & 0,000490 & 4,76 & 20 & 0,000520 & 5,86 & $6 \%$ & \\
\hline \multirow{4}{*}{11,54} & S01 & 9 & 0,000770 & 5,57 & 21 & 0,000730 & 7,44 & $-5 \%$ & \multirow{4}{*}{$-13 \%$} \\
\hline & S02 & 10 & 0,000680 & 10,25 & 22 & 0,000520 & 5,08 & $-24 \%$ & \\
\hline & S03 & 11 & 0,000370 & 27,41 & 23 & 0,000350 & 13,54 & $-5 \%$ & \\
\hline & SO4 & 12 & 0,000710 & 4,01 & 24 & 0,000570 & 4,51 & $-20 \%$ & \\
\hline & \multicolumn{9}{|c|}{ Variação da deformação no acréscimo de magnitude de carga para T1 } \\
\hline & Seção & \multicolumn{2}{|c|}{$8,27 t-10,47 t$} & \multicolumn{3}{|c|}{$8,27 t-11,54 t$} & \multicolumn{3}{|c|}{$10,47 t-11,54 t$} \\
\hline & S01 & \multicolumn{2}{|c|}{$-41,17 \%$} & \multicolumn{3}{|c|}{$13,24 \%$} & \multicolumn{3}{|c|}{$92,50 \%$} \\
\hline & S02 & \multicolumn{2}{|c|}{$163,16 \%$} & \multicolumn{3}{|c|}{$257,89 \%$} & \multicolumn{3}{|c|}{$37,16 \%$} \\
\hline & S03 & \multicolumn{2}{|c|}{$6,39 \%$} & \multicolumn{3}{|c|}{$0,73 \%$} & \multicolumn{3}{|c|}{$-5,32 \%$} \\
\hline & S04 & \multicolumn{2}{|c|}{$14,31 \%$} & \multicolumn{3}{|c|}{$65,74 \%$} & \multicolumn{3}{|c|}{$44,99 \%$} \\
\hline
\end{tabular}

Medições com valores espúrios eliminadas da análise.

Em seguida, na análise fatorial, foi confirmado que tanto o nível de temperatura $(T)$ quanto o nível de carregamento $(C)$ são diretamente proporcionais à deformabilidade do revestimento, conforme pode ser verificado no modelo fatorial com nível de confiança de $95 \%$ mostrado na 
Equação 4. Contudo, o efeito de $T$ foi superior ao de $C$ na inferência da deformação, já que seu coeficiente foi maior e o nível de significância menor. Esse comportamento foi ocasionado pela baixa rigidez do revestimento de recomposição exposto à alta irradiação solar, fazendo com que a magnitude da carga tenha efeito secundário. Neste mesmo modelo, o coeficiente linear $\left(\beta_{0}=\right.$ $558)$, o coeficiente de $T\left(\beta_{1}=119\right)$ e o coeficiente de $C\left(\beta_{2}=98\right)$ tiveram níveis de significância de $\sim 0,0 \%, 3,1 \%$ e $4,2 \%$, ou seja, menores que $\alpha=5,0 \%$, sendo estatisticamente significantes na inferência de $\varepsilon$. 0 coeficiente de T.C $\left(\beta_{3}=65\right)$ teve nível de significância de $24,8 \%$, sendo estatisticamente não significante na inferência de $\varepsilon$.

$$
\varepsilon=(558+119 . T+98 . C-65 \cdot T \cdot C) \cdot 10^{-6} ; R^{2}=0,60 .
$$

em que $\varepsilon$ :

$$
\text { deformação específica de tração [m/m]; }
$$

T: $\quad$ fator temperatura [-1 a 1]; e

C: $\quad$ fator carregamento [-1 a 1].

Através da análise de regressão fatorial, também foram identificadas medições com valores espúrios mostrados na Tabela 3, que foram excluídas para limitar os resíduos ao intervalo de \pm 0,00020 m/m. A Figura 3 apresenta dois gráficos, um referente ao modelo fatorial (a), no qual identifica-se a influência dos fatores $T$ e $C$, e o outro que apresenta os resíduos referentes aos dados de deformação validados (b), observando-se que as medições 1, 3, 7, 11, 13, 15, 19 e 23 da Tabela 3, ficaram fora da análise por apresentarem resíduos fora limite adotado.
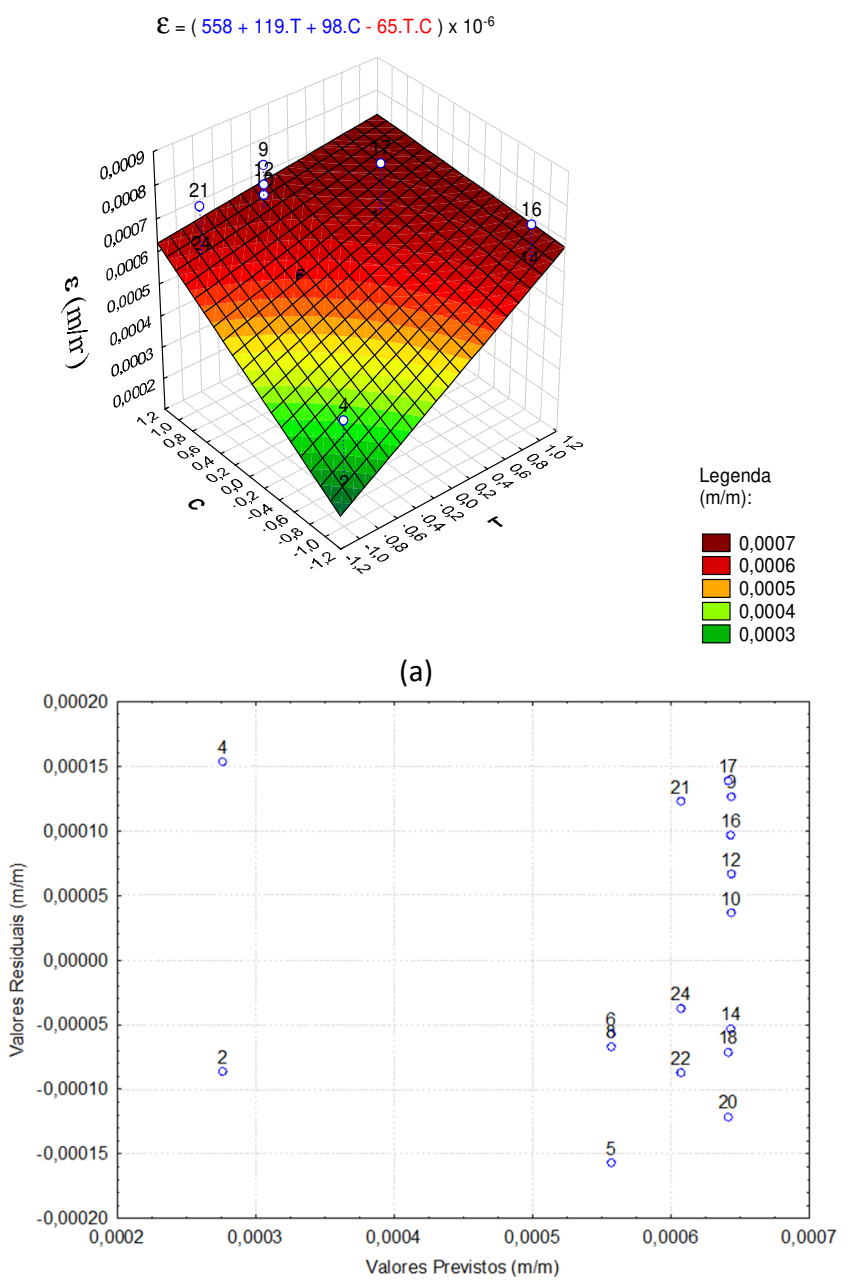

(b)

Figura 3. (a) Gráfico do modelo fatorial da equação 4; (b) Gráfico dos valores previstos versus os valores residuais. 


\subsection{Avaliações em Laboratório}

Concluídos os levantamentos de campo com a instrumentação e a posterior análise de seus dados, foi realizada caracterização mecânica dos revestimentos asfálticos, onde nos ensaios de massa específica aparente e RTI observou-se baixa rigidez nos revestimentos de recomposição das seções, obtendo-se massa específica aparente $(\gamma)$ abaixo de $2.400 \mathrm{~kg} / \mathrm{m}^{3}$ (indicada pelo fabricante do CA de recomposição) e RTI abaixo de 0,65 MPa (indicada na norma DNIT 031/2016ES). A baixa rigidez foi atribuída à compactação deficiente, que priorizou não danificar os sensores H-Gages e o cabeamento do circuito, o que gerou graus de compactação (G.C) abaixo de 90,0 \% (Tabela 4).

As baixas rigidezes foram confirmadas pelos baixos valores de módulo dinâmico $\left|\mathrm{E}^{*}\right|$ obtidos através do ensaio de RI. A Tabela 4 apresenta os parâmetros mecânicos para o revestimento de recomposição em cada seção (01, 02, 03 e 04) e também para o revestimento original (R), na temperatura padrão de $40^{\circ} \mathrm{C}$. Não foi possível obter o resultado de $\left|\mathrm{E}^{*}\right|$ para a seção 03 , devido o corpo de prova não ter suportado o ensaio em temperaturas acima de $30^{\circ} \mathrm{C}$.

Tabela 4: Parâmetros mecânicos obtidos para os revestimentos de recomposição e original

\begin{tabular}{cccccc}
\hline Revestimento & $\left|\mathrm{E}^{*}\right|(\mathrm{MPa})$ & $\boldsymbol{\mu}$ & $\mathbf{R T I}(\mathrm{MPa})$ & $\boldsymbol{\gamma}\left(\mathbf{k g} / \mathbf{m}^{\mathbf{3}}\right)$ & $\mathbf{G . C}(\%)$ \\
\hline 01 & 110 & 0,36 & 0,426 & 2070 & 86,25 \\
02 & 100 & 0,44 & 0,322 & 2020 & 84,17 \\
03 & - & - & 0,285 & 1990 & 82,92 \\
04 & 70 & 0,39 & 0,388 & 2040 & 85,00 \\
\hline $\mathrm{R}$ & 320 & 0,28 & 0,647 & 2260 & 94,17 \\
\hline
\end{tabular}

\subsection{Resultados das Análises Numéricas}

Realizada caracterização mecânica dos revestimentos, puderam-se estimar as deformações específicas de tração através dos modelos numéricos, observando diferenças significativas com os resultados de campo validados. As estimativas dos métodos numéricos, apresentadas na Tabela 5 , tiveram razoáveis diferenças em relação à instrumentação ( $\Delta$, na temperatura T1), com diferença absoluta média de $61,34 \%$, no caso do método das múltiplas camadas finitas, e $28,46 \%$ e $59,94 \%$ diferentes, no caso do modelo em elementos finitos desenvolvido, considerando atrito e desconsiderando atrito, respectivamente.

A Figura 4a apresenta o modelo geométrico do pavimento em elementos finitos e a Figura 4b o comportamento das deformações específicas longitudinais de tração na fibra inferior do revestimento asfáltico para a seção 04 , carregamento de 11,54 t e considerando aderência completa $(\mu=1,0)$, observando-se maior intensidade de deformação quanto mais próximo do centro do revestimento de recomposição, o que já era esperado, sendo neste caso igual a $0,000678 \mathrm{~m} / \mathrm{m}$.

As diferenças entre as estimativas numéricas e os valores obtidos através da instrumentação foram altas. Essas diferenças se devem principalmente às hipóteses simplificadoras dos métodos, que não representam fielmente a realidade de campo, como já falado aqui. No caso do MEF foi ainda somado à utilização de módulos elásticos linearizados e de áreas de contato pneupavimento diferentes das medidas em campo, ambos os fatores muito importantes para o comportamento mecânico do pavimento. Contudo, nas condições atribuídas à análise (sólido com comportamento elastoplástico e interface com atrito de 0,7 a 1,0), sob as condições do sistema 
(baixas rigidezes das camadas e grande magnitude de carregamento) convergiram bem para simular o problema, além de proporcionar resultados de deformações com maiores magnitudes em condições com atrito crescente. $\mathrm{Ou}$ seja, a deformação elástica sempre ocorreu adicionada às deformações plásticas que aumentavam à medida que o atrito foi ampliado, devido à consequente elevação da tensão de cisalhamento na interface.

Tabela 5: Deformações específicas de tração medidas por instrumentação (na temperatura T1) e estimadas pelos métodos numéricos e suas respectivas previsões de vida de fadiga.

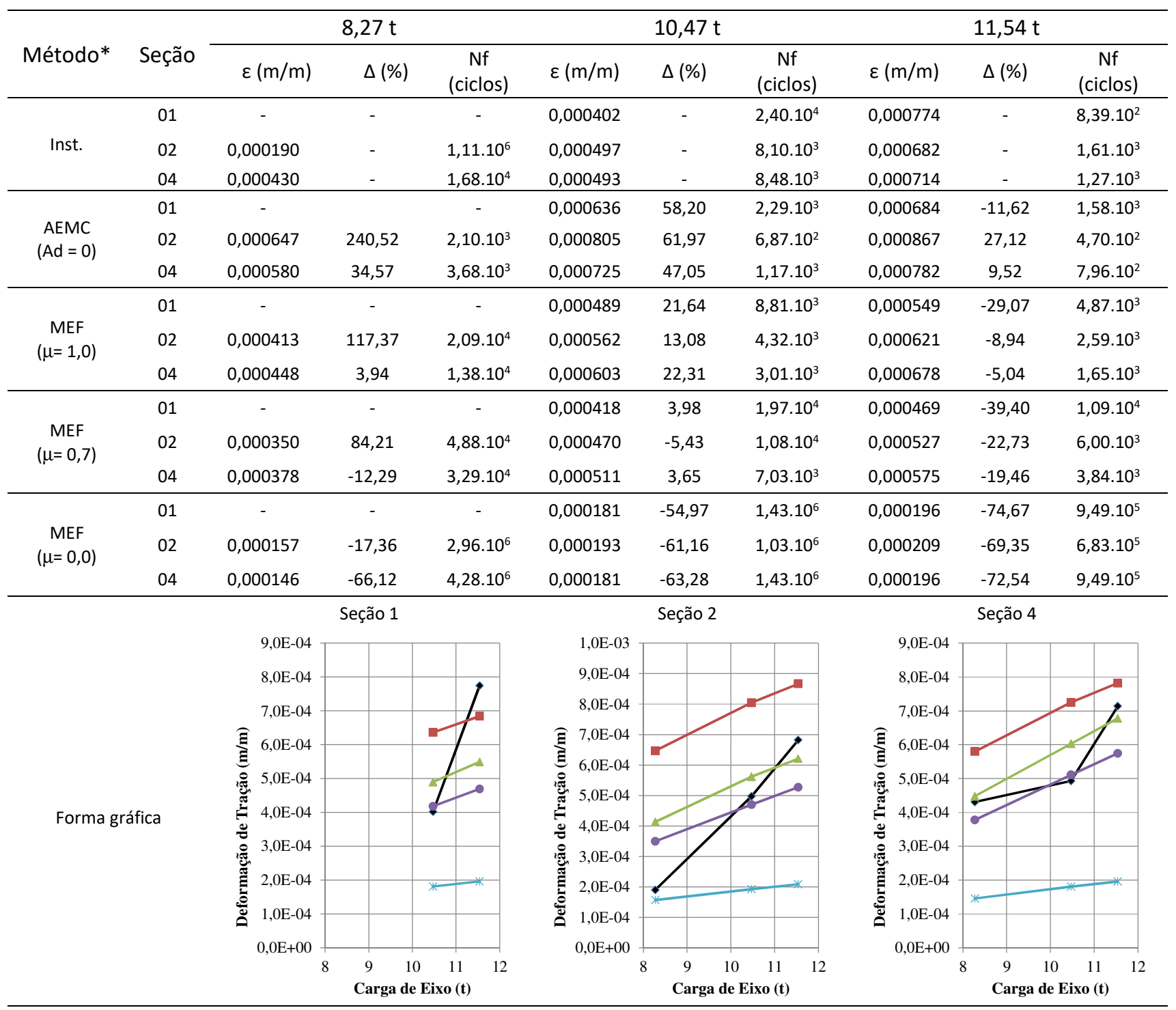

${ }^{*}$ Ad: Aderência entre camadas nas análises pelo AEMC; $\mu$ : Coeficiente de atrito considerado nas análises pelo ANSYS $®$.

Calderón e Muñoz (2015) compararam ambos os métodos e encontraram diferenças em torno de 3,00\%. No caso deste trabalho, foi -35,72\% e -74,04\% comparando as estimativas com o MEF com e sem atrito, respectivamente, com o método das múltiplas camadas finitas. É difícil comparar e avaliar modelos em elementos finitos desenvolvidos em trabalhos diferentes, pois, pelo fato do método ser aberto a várias possibilidades de modelagem de condições de contorno, comportamento mecânico dos materiais e composição do pavimento, cada modelo é único, podendo ser melhorado e se aproximar mais das condições de campo. 


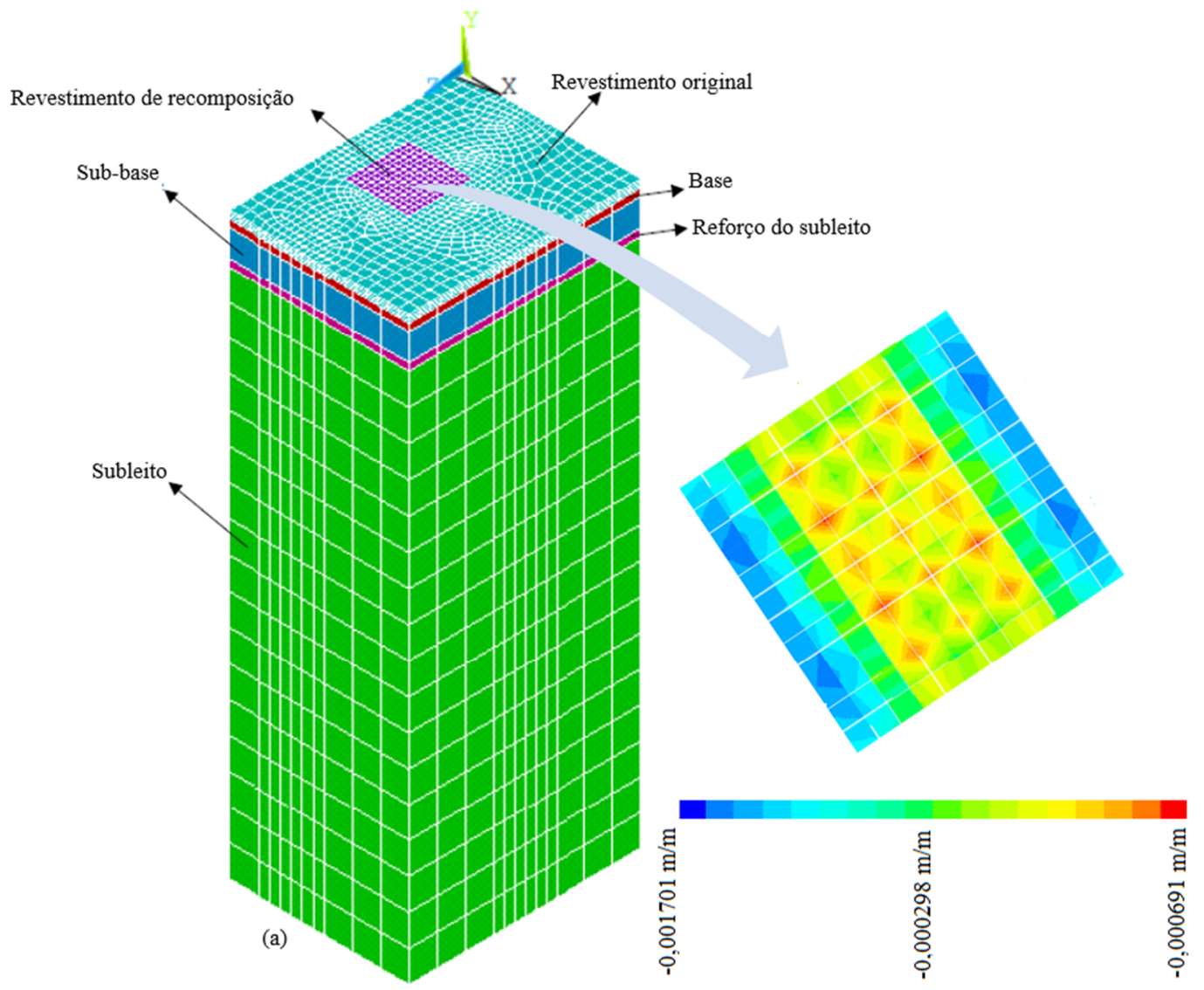

Figura 4. (a) Modelo geométrico em elementos finitos; (b) Comportamento das deformações na fibra inferior do revestimento de recomposição.

\subsection{Resultados das Análises de Vida de Fadiga}

No comparativo entre as previsões de vida fadiga (Nf), baseadas nos resultados das análises numéricas e nas previsões a partir dos dados da instrumentação, as diferenças de número de ciclos de carregamento para atingir a fadiga do revestimento foram bem significativas, conforme apresentado na Tabela 5 . As diferenças médias foram de -58,21\% para o método das camadas finitas, 35,17\% para o MEF considerando o atrito pleno, e 363 vezes superior desconsiderando o atrito, valor considerado inaceitável, mostrando grande importância da consideração do atrito/aderência no MEF. Além do método das camadas finitas fornecer previsão de fadiga mais próxima da calculada com os dados da instrumentação, que foram tomados como referência, esse método apresentou tendência ao superdimensionamento, o que é de certa forma preferível à tendência ao subdimensionamento, apresentada no MEF.

\section{CONCLUSÕES}

Após análise dos resultados de campo, embora o carregamento e a temperatura influenciem na magnitude das deformações, essa última foi estatisticamente mais significante, especialmente nas menores magnitudes de carga e altas temperaturas registradas na análise. Pode ter sido consequência da baixa rigidez dos revestimentos, que tiveram deficiência na compactação, o que também impossibilitou concluir sobre o principal fator que influencia nas deformações em pavimentos com condições de compactação típicas.

Quanto à análise da eficácia dos métodos numéricos estudados, acredita-se que os mesmos deveriam ser capazes de replicar as condições observadas em campo, independentemente da 
qualidade dos materiais aplicados, fornecendo resultados de deformações semelhantes às captadas pela instrumentação. Neste contexto, algumas constatações podem ser elencadas com base nos resultados e análises estatísticas realizadas:

- O contato de forma retangular entre o pneu e pavimento fornece resultados bem distintos aos observados com forma circular (tradicionalmente aplicada em análises de tensões e deformações em pavimentos), tanto na forma quanto a magnitude de distribuição do carregamento;

- As condições de aderência ou atrito entre camadas devem ser consideradas para a interface entre materiais que apresentem alguma cimentação (asfáltica ou hidráulica), trazendo substancial influência na análise numérica, sobretudo para o método de elementos finitos. Já na interface com algum material que não a possua, as condições de aderência ou atrito podem ser desprezadas;

- Como esperado, a análise numérica considerando os valores de módulo dinâmico para as misturas asfálticas, na mesma temperatura e frequência de carregamento das observações com instrumentação em campo, conduz a resultados mais próximos à realidade que com uso do módulo de resiliência a $25^{\circ}$ c e $1 \mathrm{~Hz}$;

- No método analítico de Múltiplas Camadas Elásticas, os erros (diferença dos resultados da simulação numérica para os dados monitorados por instrumentação) diminuem com o aumento do carregamento aplicado ao pavimento, que no caso deste trabalho foram de $8,27 \mathrm{t}, 10,47$ t e $11,54 \mathrm{t}$;

- Quando da aplicação de análise pelo Método de Elementos Finitos sem a consideração de atrito entre as camadas, o erro cresce com o aumento do carregamento aplicado pelo eixo do veículo ao pavimento; e

- Na análise com o Método de Elementos Finitos considerando o atrito entre camadas (especialmente para $\mu=0,7)$, os erros observados foram praticamente estáveis e os menores entre os tipos de análise realizados, sendo observada ainda tendência a erros ainda menores com o carregamento de 10,47 t, próximo à carga legal do eixo simples de rodas duplas.

A análise de tensões e deformações em pavimentos com o Método de Elementos Finitos, considerando atrito na interface entre camadas cimentadas, demonstra uma tendência à obtenção de resultados aproximados aos de campo. Contudo, algumas observações adicionais podem ser indicadas para melhorar a eficácia deste método, de modo que se possa aprimorar para retroanalisar à situação observada na instrumentação (campo), como usar modelos constitutivos que traduzam melhor as condições dos módulos elásticos dos materiais das camadas que sejam dependentes do estado de tensões, carregamentos nas mesmas áreas de contato e frequência observadas em campo, e temperatura igual à do momento dos ensaios em campo, de forma a inferir melhor o módulo dinâmico da mistura asfáltica.

\section{AGRADECIMENTOS}

Ao CNPq pelo apoio financeiro à realização desta pesquisa, a partir de projeto contemplado no Edital MCTI/CNPQ/Universal $14 / 2014$.

\section{REFERÊNCIAS}

Abu-Farsakh, M.; J. Gu; G. Voyuadjis e Q. Chen (2014) Mechanical-empirical Analysis of the Results of Finite Element Analysis on Flexible Pavement with Geogrid Base Reinforcement. Internacional Journal of Pavement Engineering, v. 15, n. 9, p. 786798. DOI: $10.1080 / 10298436.2014 .893315$. 
ASTM (2015) ASTM E1876-09: Standard Test Method for Dynamic Young's Modulus, Shear Modulus, and Poisson's Ratio by Impulse Excitation of Vibration. American Society for Testing and Materials, West Conshohocken, PA.

Balbo, J. T. (2007) Pavimentação Asfáltica: materiais, projeto e restauração. Oficina de Textos, 588 p.

Bastos, J. B. dos (2016) Considerações sobre a Deformação Permanente de Pavimentos Asfálticos no Dimensionamento Mecanístico-Empírico. Tese (doutorado). Programa de Pós-Graduação em Engenharia de Transportes, Universidade Federal do Ceará, Fortaleza, CE.

Beskou, N. D.; S. V. Tsinopoulos e D. D. Theodorakopoulos (2015) Dynamic Elastic Analysis of 3-D Flexible Pavements under Moving Vehicles: A unified FEM treatment. Soil Dynamics and Earthquake Engineering, v. 82, p. 63-72. DOI: 10.1016/j.soildyn.2015.11.013.

Bernucci, L. B.; L. M. G. da Motta; J. A. P. Ceratti e J. B. Soares (2010) Pavimentação Asfáltica: Formação Básica para Engenheiros. Associação Brasileira das Empresas Distribuidoras de Asfalto, Rio de Janeiro, RJ.

Boussinesq, J. (1885) Application des Potentiels a l'étude de l'equilibreet du Mouvement des Solids Elastiques, Gauthier-Villars, Paris.

Burmister, D. M. (1945a) The General Theory of Stresses and Displacements in Layered Systems I. Journal of Applied Physics, v. 16, n. 2, p. 89-94. DOI: 10.1063/1.1707558.

Burmister, D. M. (1945b) The General Theory of Stresses and Displacements in Layered Soil Systems II. Journal of Applied Physics, v. 16, n. 3, p. 126-127. DOI: 10.1063/1.1707562.

Calderón, W. R. e M. R. P. Muños (2015) Three-dimensional Modeling of Pavement with Dual Load Using Finite Element. Dyna, v. 82, n. 189, p. 30-38. DOI: 10.15446/dyna.v82n189.41872.

CONTRAN (1998) Resolução no 12, de 06 de fevereiro de 1998. Conselho Nacional de Trânsito, Brasília, DF.

CONTRAN (2015) Resolução nº 526, de 20 de abril de 2015. Conselho Nacional de Trânsito, Brasília/DF.

Costa, G. M. (2017) Método de ressonância por impacto: Obtenção de curvas mestras de módulo dinâmico e ângulo de fase em misturas asfálticas brasileiras. Dissertação (mestrado). Programa de Pós-Graduação em Engenharia Civil, Universidade Federal de Sergipe, São Cristóvão, SE.

Deusen, D. A. V; D. E. Newcomb e J. F. Labuz (1992) A Review of Instrumentation Technology for the Minnesota Road Research Project. Research Administration and Development Section Office of Materials and Research Minnesota Department of Transportation. Minnesota.

DNIT (2006) DNIT. 031/2006 - ES. Pavimentos Flexíveis - Concreto asfáltico - Especificação de Serviço. Diretoria de Planejamento e Pesquisa, Departamento Nacional de Infraestrutura de Transportes, Rio de Janeiro, RJ.

DNIT (2008) Identificação de Sistemas de Pesagem em Movimento: Projeto de Instrumentação para Medição de Deformação do Pavimento. Departamento Nacional de Infraestrutura de Transportes, Santa Catarina.

Dong, Z.; Y. Tan; S. Li e L. Cao (2012) Rutting Mechanism Analysis of Heavy-duty Asphalt Pavement Based on Pavement Survey, Finite Element Simulation, and Instrumentation. Journal of Testing and Evaluation, v. 40, n. 7, p. 1-10. DOI: 10.1520/JTE20120162.

Elseifi, M. A.; M. A. Mohammad e Z. J. Zhang (2012) Assessment of Stress and Strain Instrumentation in Accelerated-Pavement Testing. International Journal of Pavement Research and Technology, v. 5, n. 2, p. 121-127. DOI: 10.6135/ijprt.org.tw/2012.5(2).121.

Franco, F. A. (2007) Método de Dimensionamento Mecanístico-Empírico de Pavimentos Asfálticos: Sispav. Tese (doutorado). COPPE, Universidade Federal do Rio de Janeiro, Rio de Janeiro, RJ.

Fritzen, M. A. (2016) Desenvolvimento e Validação de Função de Transferência para Previsão do Dano por Fadiga em Pavimentos Asfálticos. Tese (doutorado). COPPE, Universidade Federal do Rio de Janeiro, Rio de Janeiro, RJ.

Gonçalves, F. P. (2002) Estudo do Desempenho de Pavimentos Flexíveis a Partir de Instrumentação e Ensaios Acelerados. Tese (doutorado). Programa de Pós-Graduação em Engenharia Civil, UFRGS, Porto Alegre.

Holanda, A. S.; E. Parente Jr.; T. D. P. Araújo; L. T. B. Melo; F. Evangelista Jr. e J. B. Soares (2006) Finite Element Modeling of Flexible Pavements. In: Iberian Latin- American Congress on Computational Methods in Engineering (CILAMCE), Anais. Belém, PA.

Ioannides, A. M. e L. Khazanovich (1998) General formulation for multilayered pavement systems. Journal of Transportation Engineering, v. 124, n. 1, p. 82-90. DOI: 10.1061/(ASCE)0733-947X(1998)124:1(82).

Kim, Y. R.; C. Baek; B. S. Underwood; V. Subramanian; M. N. Guddati e K. Lee. (2008) Application of Viscoelastic Continuum Damage Model Based Finite Element Analysis to Predict the Fatigue Performance of Asphalt Pavements. KSCE Journal of Civil Engineering, v. 12, p. 109-120. DOI: 10.1007/s12205-008-0109-x.

Kyowa (2011) Strain Gages. KYOWA Eletronic Instruments CO LTD.

Leiva-Villacorta, F. e D. H. Timm (2012) Simulating the effects of instrumentation on measured pavement response. In: Jones, D.; J. Harvey; A. Mateos e I. Al-Qadi. Advances in Pavement Design through Full-scale Accelerated Pavement Testing. Taylor \& Francis Group, p. 153-161. DOI: 10.1201/b13000-23.

Li, Y.; E. Onodera e A. Chiba (2010) Friction Coefficient in Hot Compression of Cylindrical Sample. Materials Transactions, v. 51, n. 7, p. 1210-1215. DOI: 10.2320/matertrans.M2010056.

Madenci, E. e I. Guven (2006) The Finite Element Method and Applications in Engineering Using ANSYS®. Springer.

Matos, L. J. S. (2015) Análise de Tensões Verticais em Estrutura de Pavimento Semirrígido Instrumentado Sob Carregamento Variado. Dissertação (mestrado). Programa de Pós-Graduação em Engenharia Civil, Universidade Federal de Sergipe, São Cristóvão, SE. 
Nascimento, L. A. H. (2015) Implementation and Validation of the Viscoelastic Continuum Damage Theory for Asphalt Mixture and Pavement Analysis in Brazil. North Carolina State University.

Nakasone, Y.; S. Yoshimoto e T. A. Stolarski (2006) Engineering Analysis with ANSYS Software. Butterworth-Heinemann.

NCHRP/TRB (2004) Guide for Mechanistic-Empirical Design of New and Rehabilitated Pavement Structures, Appendix RR: Finite Element Procedures for Flexible Pavement Analysis. Illinois.

Pelletier, H.; J. Krierb e C. Gauthiera (2011) Influence of local friction coefficient and strain hardening on the scratch resistance of polymeric surfaces investigated by finite element modeling. Procedia Engineering, v. 10, p. 1772-1778. DOI: 10.1016/j.proeng.2011.04.295.

Saevarsdottir, T. e S. Erlingsson (2016) Deformation Modelling of Instrumented Flexible Pavement Structure. Procedia Engineering, v. 143, p. 937-944. DOI: 10.1016/j.proeng.2016.06.076.

SAPEM (2014) South African Pavement Engineering Manual. The South African National Roads Agency Ltd.

Silva, S. de A. T. e; J. B. dos S. Bastos e J. B. Soares (2015) Influência da aderência na análise de pavimentos asfálticos. In: 44aㅗ Reunião Anual de Pavimentação, 18ํㅡㄹ Eontro Nacional de Conservação Rodoviária, Anais. Foz do Iguaçu, PR.

Souza, F. V. e J. B. Soares (2003) Considerações sobre Módulo Resiliente e Módulo Dinâmico em Misturas Asfálticas com Base na Teoria da Viscoelasticidade. In: Congresso Ibero-Latinoamericano do Asfalto, Anais, Quito.

Teixeira, V. F; F. V. de Souza e J. B. Soares (2007) Modelagem da Vida de Fadiga e do Acúmulo de Deformações Permanentes em Pavimentos Asfálticos por Meio de um Modelo de Dano Contínuo. Revista Transportes, v. 15, n. 2. DOI: 10.14295/transportes.v15i2.32.

Trzepieciński, T. e H. G. Lemu (2015) Proposal for an Experimental-Numerical Method for Friction Description in Sheet Metal Forming. Journal of Mechanical Engineering, v. 61(2015)6, p. 383-391. DOI: 10.5545/sv-jme.2015.2404.

Yoder, E. J. e M. W. Witczak, (1975) Principles of pavement design. John Wiley \& Sons, New York, NY. 